\title{
Logic functions based on optical bias controlled SiC tandem devices
}

\author{
V. Silva ${ }^{1,2}$, M. A. Vieira ${ }^{1,3}$, M. Vieira ${ }^{*, 1,2}$, P. Louro ${ }^{1,2}$, A. Fantoni $^{1,2}$, and M. Barata ${ }^{1,2}$ \\ ${ }^{1}$ Electronics Telecommunication and Computer Department, ISEL, R. Conselheiro Emídio Navarro, 1949-014 Lisboa, Portugal \\ ${ }^{2}$ CTS-UNINOVA, Quinta da Torre, Monte da Caparica, 2829-516 Caparica, Portugal \\ ${ }^{3}$ DEE-FCT-UNL, Quinta da Torre, Monte da Caparica, 2829-516 Caparica, Portugal
}

Received 12 August 2013, revised 7 December 2013, accepted 7 January 2014

Published online 7 February 2014

Keywords optoelectronics, logical functions, digital light signals, a-SiC sensor

* Corresponding author: e-mail mv@isel.ipl.pt, Phone: +351 218317290 , Fax: +351 218317114

The purpose of this paper is the design of an optoelectronic circuit based on a-SiC technology, able to act simultaneously as a 4-bit binary encoder or a binary decoder in a 4-to-16 line configurations and show multiplexer-based logical functions. The device consists of a p-i'(a-SiC:H)-n/p-i(a-Si:H)-n multilayered structure produced by PECVD. To analyze it under informationmodulated wave (color channels) and uniform irradiation (background) four monochromatic pulsed lights (input channels): red, green, blue and violet shine on the device. Steady state optical bias was superimposed separately from the front and the back sides, and the generated photocurrent was measured.
Results show that the devices, under appropriate optical bias, act as reconfigurable active filters that allow optical switching and optoelectronic logic functions development providing the possibility for selective removal of useless wavelengths. The logic functions needed to construct any other complex logic functions are the NOT, and both or either an AND or an OR. Any other complex logic function that might be found can also be used as building blocks to achieve the functions needed for the retrieval of channels within the WDM communication link.
1 Introduction Multi-disciplinary technologies for telecommunication systems have evolved from material systems for LEDs and lasers sources to optical fiber technology and photo detection materials. Newly developed technologies, for infrared telecommunication systems, allowed increase of capacity, distance and functionality. Far beyond traditional applications in optical interconnects at telecommunications wavelengths, the $\mathrm{SiC}$ nanophotonic integrated circuit platform has recently proven its merits for working with visible range optical signals $[1,2,3]$.

In order to enhance the transmission capacity and the application flexibility of optical communication and sensor systems, associated efforts have to be considered, namely: Wavelength Division Multiplexing (WDM) based on amorphous technology $[4,5,6,7,8,9,10]$ when different optical signals are encoded in the same optical transmission path; the IP-based multimedia services architecture to provide transparent communication over different networks $[11,12]$, switching and control with the design of new reconfigurable logic active filter gates by "bridging the gaps" and combining the optical filters properties [13, 14].

The SiC tuneable background filters are used to perform several filtering processes and then different signal processing functions: amplification, switching, and wavelength conversion [15]. In this paper the $\mathrm{SiC}$ tunable background filters form the bridge between the infrared and visible windows. The device can be used as a bistable optical gate acting either as a short- or a long- pass band filter or as a band-stop filter, depending on the wavelength of the external background. In consequence it offers the opportunity to provide alternative and additional low cost services to improve operative production processes in office, home and automotive networks.

Recovery of individual channels transmitted in the visible spectrum in open space can be successful with optoelectronic devices that interface a signal processing system. We propose the use of a multilayered structure based 
on amorphous silicon technology which can be reconfigured to perform WDM optoelectronic logic functions [16, 17]. The multilayered structure will be a solution in WDM technique for information transmission and decoding in the visible range [18]. The operating principle exploits the physical properties of the nonlinear device to perform logic functions. The logic functions needed to construct any other complex logic functions are the NOT, and both or either an AND or an OR $[19,20]$. Any other complex logic function that might be found can also be used as building blocks to achieve the functions needed for the retrieval of channels within the WDM communication link. Here we present some logical functions that can be configured through this device.

2 Device deposition and operation The optoelectronic device consists of a p-i'(a-SiC:H)-n/p-i(a-Si:H)-n hetero structure produced by PECVD as displayed in Fig. 1. The thickness $(200 \mathrm{~nm})$ and the optical gap $(2.1 \mathrm{eV})$ of the a-SiC:H intrinsic layer (i'-) are optimized for blue collection and red transmittance and the thickness $(1000 \mathrm{~nm})$ of a-Si:H one (i-) adjusted to achieve full absorption in the green and high collection in the red spectral range. As a result, both front and back diodes act as optical filters confining, respectively, the blue and the red optical carriers, while the green ones are absorbed across both. The optoelectronic characterization and device deposition is described elsewhere $[1,2]$. Separate monochromatic pulsed lights, $\left(\lambda_{\mathrm{R}}=626 \mathrm{~nm} ; \lambda_{\mathrm{G}}=526 \mathrm{~nm}, \lambda_{\mathrm{B}}=470 \mathrm{~nm}, \lambda_{\mathrm{V}}=400 \mathrm{~nm}\right)$ act as input channels. This polychromatic mixture (multiplexed signal) illuminates the device from its front side.

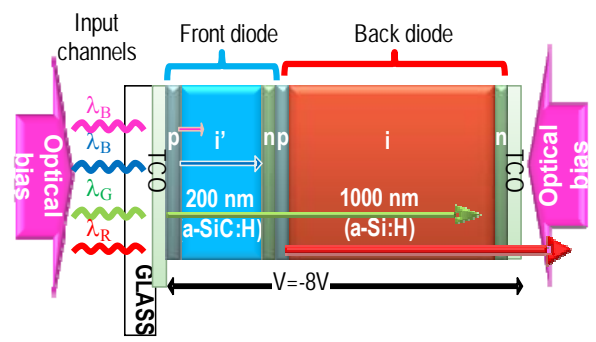

Figure 1 Device configuration and operation.

Tuning of each channel is performed by steady state violet optical bias $\left(\phi_{\text {bias }}=2800 \mu \mathrm{W} / \mathrm{cm}^{2}\right)$ superimposed either from the front or back side of the device. Input signals are synchronous square waves, in different bit sequences, which modulate the light of ultra-bright LEDs: red (R; $\left.28 \mu \mathrm{W} / \mathrm{cm}^{2}\right)$, green $\left(\mathrm{G} ; 22 \mu \mathrm{W} / \mathrm{cm}^{2}\right)$, blue $\left(\mathrm{B} ; 30 \mu \mathrm{W} / \mathrm{cm}^{2}\right)$ and violet $\left(\mathrm{V} ; 78 \mu \mathrm{W} / \mathrm{cm}^{2}\right)$.

When an external optical bias is applied to a double pin structure, its main influence is in the field distribution within the less photo excited sub-cell [21].The front cell, under long wavelength irradiation; the back cell, under short wavelength light and both, under medium steady state illumination. In comparison with thermodynamic equilibrium conditions (dark), the electric field under illu- mination is lowered in the most absorbing cell (self forward bias effect) while the less absorbing reacts by assuming a reverse bias configuration (self reverse bias effect).

So, front violet optical bias enhances the spectral sensitivity in the long wavelength ranges and quenches in the short wavelength range. The back bias has an opposite behavior; it reduces the collection in red/green wavelength ranges and amplifies the blue/violet one. The sensor is a bias wavelength current-controlled device that makes use of changes in the wavelength of the background to control the power delivered to the load, acting as an optical amplifier. Its gain depends on the background wavelength and side that controls the electrical field profile across the device. If the electrical field increases locally (self optical amplification) the collection is enhanced and the gain is higher than one. If the field is reduced (self optical quench) the collection is reduced and the gain is lower than one. This optical nonlinearity makes the transducer attractive for optical communications and can be used to distinguish a wavelength, to read a color image, to amplify or to suppress a color channel or to multiplex or demultiplex an information-modulated wave. It provides a relatively flat partial gain spectrum which makes it possible to use multiple wavelengths. It will be an indispensable element of next generation WDM systems in the visible range [17].

3 Optical bias controlled filters Illuminating the device with violet light and different intensities, either at
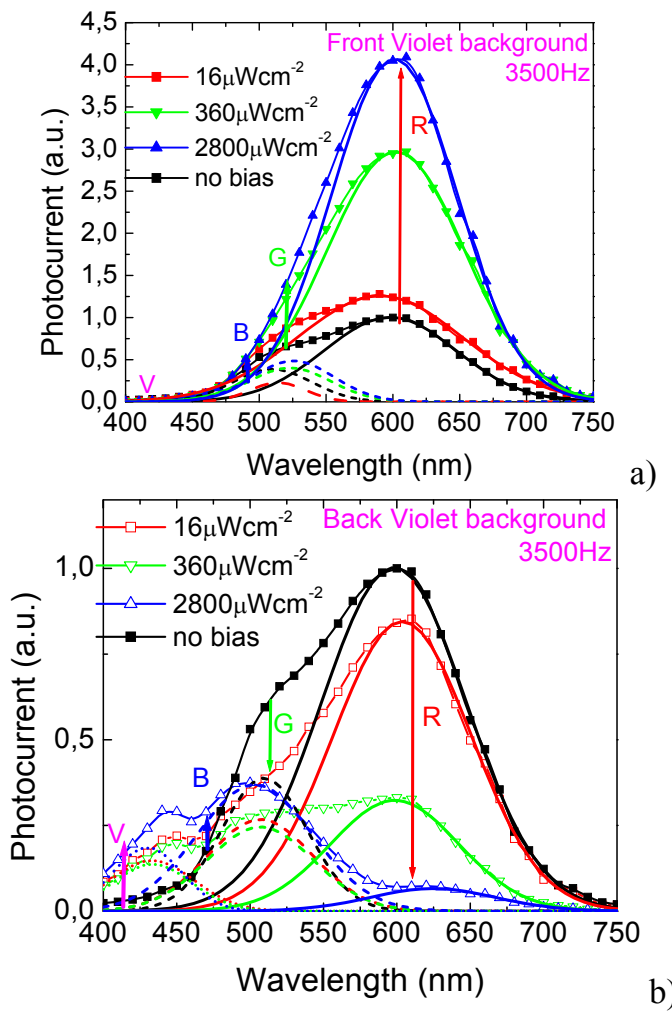

Figure 2 Normalized spectral photocurrent under front (a) and back (b) violet irradiations with different intensities. 
front or at the back side, changes the spectral photocurrent changes as pictured in Fig. 2.

For comparison, both graphics show the spectral photocurrent without applied optical bias (dark). When violet irradiation is applied from the front side (Fig. 2a) or from the back (Fig. 2b) the spectral response changes significantly. A peak fit adjustment to the data was performed (lines) with peaks centered on $630 \mathrm{~nm}$ (solid), $520 \mathrm{~nm}$ (dashed) and $430 \mathrm{~nm}$ (dotted).

Results show that under increased intensity and front violet irradiation the peak centered at $630 \mathrm{~nm}$ (red range) strongly increases whereas the blue part of the spectrum is negligible. Illuminating the back side of the device it quenches the $630 \mathrm{~nm}$ peak intensity and increases the blue range mainly at the three marked positions. So, front illumination and high intensity enhances the reddish part of the spectrum while under back illumination the main enhancement occurs in the violet-blue region. A trade-off between the background intensity and the enhancement or quenching of the different spectral regions has to be taken into account.

Plotting the ratio between the spectral responses with and without optical bias $\left(2300 \mu \mathrm{Wcm}^{-2}\right)$ either from the front or back gives the spectral gain $\left(\alpha^{\mathrm{V}}\right)$ presented in Fig. 3. As expected from Fig. 2, under back bias the gain is high at short wavelengths and lowers strongly at wavelengths higher than $500 \mathrm{~nm}$, acting as a short-pass filter. Under violet front light the device works as a long-pass filter for wavelengths higher than $550 \mathrm{~nm}$, blocking shorter wavelengths.

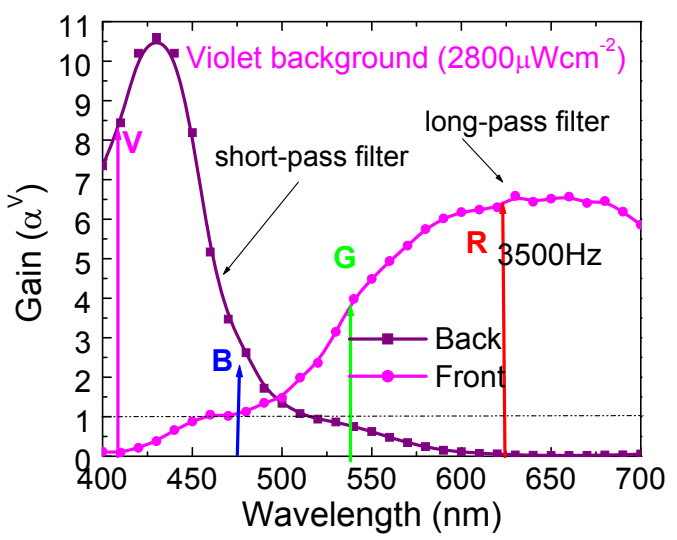

Figure 3 Normalized spectral photocurrent under front and back violet irradiations.

Results show that by combining the irradiation sides the short-, and long- spectral region can be sequentially tuned. The medium region can only be tuned by using both active filters

Four monochromatic pulsed lights, separately (R, G, B and $\mathrm{V}$ input channels) illuminated the device at $12000 \mathrm{bps}$. Steady state violet optical bias was superimposed separately from the front (Fig. 4a) and the back (Fig. 4b) sides and without it and the photocurrent measured at $-8 \mathrm{~V}$. The signals were normalized to their values without applied background. The optical gain (amplification factor) for the four individual channels $\left(\alpha^{\mathrm{V}}, \mathrm{G}, \mathrm{B}, \mathrm{V}\right)$ under front and back irradiation are also displayed.

Results show that, even under transient conditions and using commercial LEDs as pulsed light sources, the background side affects the signal magnitude of the color channels. As shown in Fig. 2 and Fig. 3, under front irradiation, it enhances mainly the spectral sensitivity in the mediumlong wavelength ranges $\left(\alpha_{\mathrm{R}}=4.7, \alpha_{\mathrm{G}}=2.4\right)$. Violet radiation is absorbed at the top of the front diode, increasing the electric field at the back diode [22] where the red and part of the green incoming photons are absorbed (see Fig. 1). Under back irradiation the electric field increases mainly near the front $p-i$ ' interface where the violet and part of the blue incoming channels generate most of the photocarriers $\left(\alpha^{\mathrm{V}}{ }_{\mathrm{V}}=11, \alpha_{\mathrm{B}}^{\mathrm{V}}=1.5\right)$. So, by switching between front to back irradiation the photonic function is modified from a longto a short-pass filter allowing, alternately selecting the red or the violet channels.

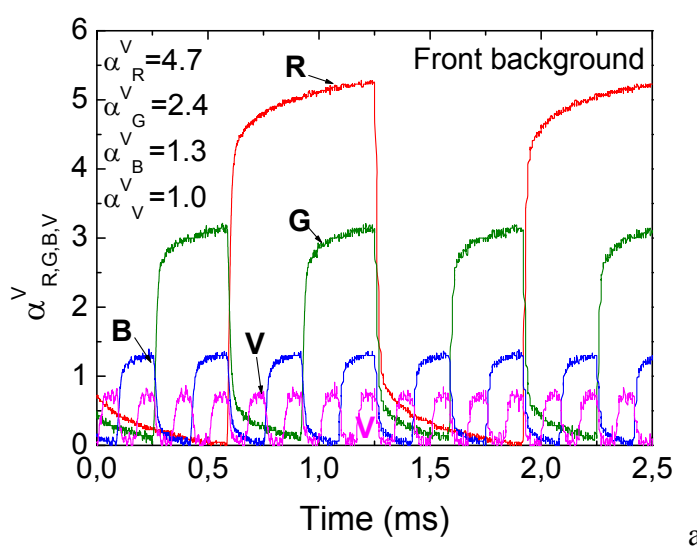

a)

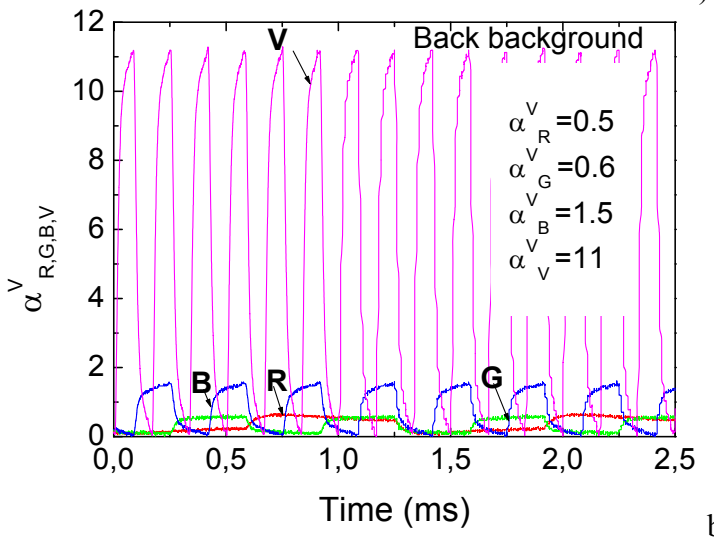

b)

Figure 4 Red (R), green (G), blue (B) and violet (V) input channels, under violet optical bias applied from the front (a) and from the back (b) sides and normalized to their values without background.

4 Encoder/decoder Four combined monochromatic pulsed lights (multiplexed signal, Fig. 5) illuminated the device at 12000 bps. Steady state violet optical bias was 
superimposed separately from the front and the back sides and the photocurrent was measured.

Results show that, even under transient conditions and in the presence of several channels simultaneouly, the side of the background affects the form and the magnitude of the MUX signal in opposite ways [23]. Under front and back irradiation, sixteen levels $\left(2^{4}\right)$ are detected and grouped into different subgroups due to the different gains of the same color channel under front and back irradiations (see arrow in Fig. 3 and Fig. 4). The dash-dot line is the threshold for both. Under front irradiation the eight upper levels are ascribed to the presence of the red channel ON and the lowers to its absence, while under back irradiation the higher eight correspond to the presence of the violet channel ON and the lower to its lack. This allows, respectively, the decoding of the red and violet channels (8-to-1 multiplexer; long- and short-pass filter functions). As under front irradiation the green channel is amplified $\left(\alpha_{\mathrm{G}}^{\mathrm{V}}>1\right)$, the blue channel stays in its value, and the violet is almost suppressed. Under back illumination the blue channel is enhanced and the green and red are reduced $\left(\alpha_{\mathrm{B}}^{\mathrm{V}}>1\right.$ and $\alpha_{\mathrm{R}}^{\mathrm{V}}$ and $\left.\alpha_{\mathrm{G}^{\mathrm{V}}}<1\right)$. It is then possible to ascribe a specific weight assigned to each bit position [24]. Due to the different optical gains (Fig. 4), the selection index for those ordered 16-element is a 4-bit binary [RGBV] code under front irradiation or a 4-bit binary [VBGR] under violet illumination. The multiplexer select code represents an address or index, into the sixteen ordered inputs.

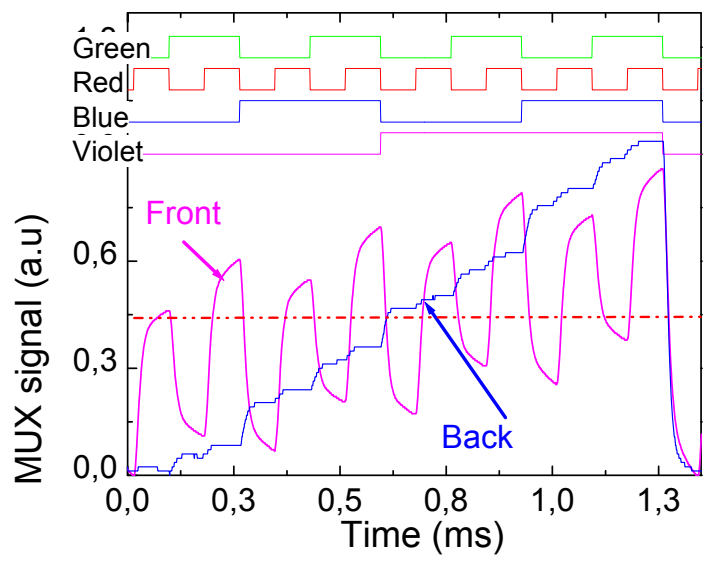

Figure 5 Multiplexed signal under front and back violet irradiation. On the top the signals used to drive the input channels are shown to guide the eyes into the ON/OFF channel states.

5 Digital light signals There are three basic logical functions or operations: AND (conjunction), OR (disjunction) and NOT (inverse). It is clear from Fig. 5 that by using only two long and short wavelengths channels (the red and blue or the green and violet) an inverter can be built and controlled by the violet irradiation either at the front or back side of the device. Here, the true (T) or false (F) outcome is correlated to the relationship between the difference of both red and blue or green and violet components of the input signals Using visible light to mimic the logic values that input the operations and their result, implies that the value $T$ is the presence of light and the value $F$ its absence. The inverse function must block the presence of light $(\mathrm{T}->\mathrm{F})$ and when there is no light it has to emit it $(\mathrm{F}->\mathrm{T})$.

This in the pi'npin device implies another approach. The sensor works as tuneable background filter (see Fig. 3 and Fig. 4). By defining the presence of a signal in one bandwidth as the $\mathrm{T}$ value and as $\mathrm{F}$ on the other it is possible to define the inverse function of an input signal if that signal is composed by those two wavelengths. Here, the presence of a signal in the long-pass bandwidth was considered as a T logical value.

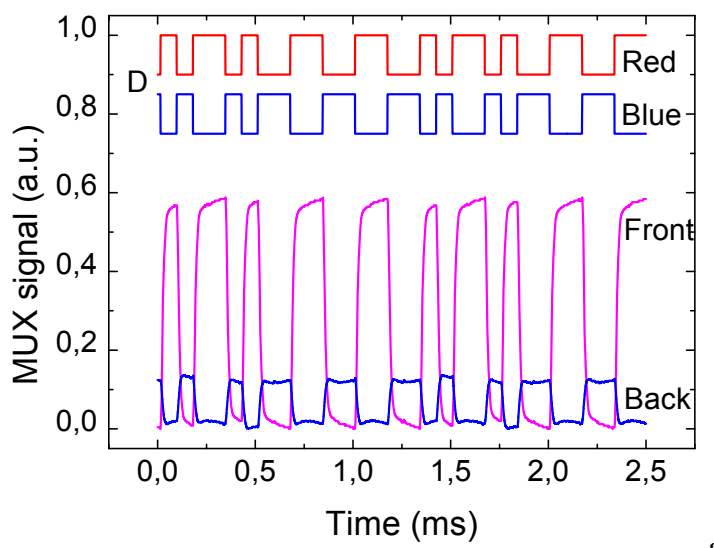

a)

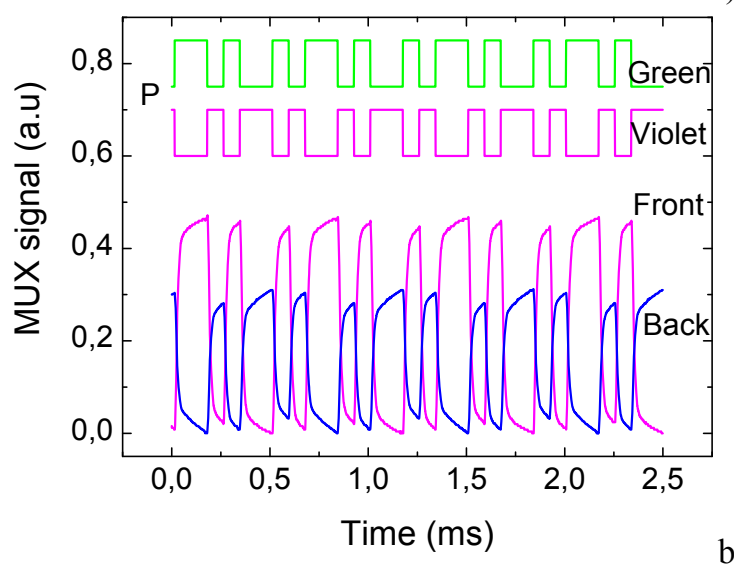

b)

Figure 6 Two examples of digital light signals and its MUX signals under front and back irradiation. On the top the signals used to drive the input channels are shown to guide the eyes into the ON/OFF channel states.

So, a visible digital light signal, D, is then the multiplex signal of two different wavelength channels, in the long and in the short wavelength ranges, under front and back violet irradiation, henceforth represented as $\mathrm{D}[\mathrm{L}, \mathrm{S}]$ where $\mathrm{L}$ and $\mathrm{S}$ represent the channel color.

As an application of Fig. 5, in Fig. 6 there are examples of two possible digital light signals: D[R, B] (a) and $\mathrm{P}[\mathrm{G}, \mathrm{V}]$ (b). Results show that by changing the background side at an appropriate intensity and wavelengths the digital 
light signal can be obtained allowing describing, in the visible range, the basic logic functions.

Some examples of different logic gates will be present in the following text as one of the the device possible applications.

5.1 NOT gate It is clear from Fig. 6 that by using only two long and short wavelengths channels (the red and blue or the green and violet) an inverter can be built and controlled by the violet irradiation either at the front or back side of the device. Here, the true or false outcome is correlated to the relationship between the difference of both red and blue or green and violet components of the input signals. Figure 7 shows a NOT function built with the use of a multiplexer, in a) the output signal is the inverse of $\mathrm{A}$ and in $b$ ) invert selects either $B$ or $\sim B$ as the output, where $\sim B$ is the inverse of $\mathrm{B}$. In the following examples 1 is the value $\mathrm{T}$ and 0 the value $\mathrm{F}$.

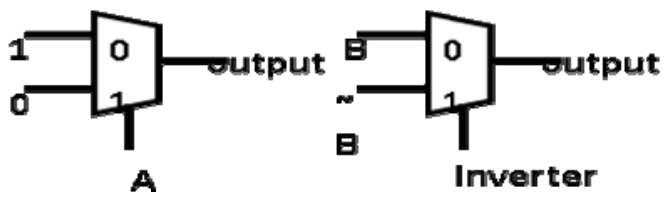

a)

b)

Figure 7 Two circuits using a multiplexer as a NOT logical function.

Equations (1) and (2) show, respectively, for the circuit in Fig. 7a and Fig. $7 \mathrm{~b}$ the corresponding transformations:

$$
\begin{aligned}
& \text { output }=1 \cdot \sim A+0 \cdot A=\sim A, \\
& \text { output }=B \cdot \sim \text { Invert }+\sim B \cdot \text { Invert }=B \oplus \text { Invert } .
\end{aligned}
$$

Equation (2) can be simplified as the XOR (exclusive $\mathrm{OR})$ relation between $\mathrm{B}$ and invert. If Invert is 0 then the output is $\mathrm{B}$ otherwise the output is $\sim \mathrm{B}$.

Here, the digital light signal components are synchronized and differential; when one is on the other is off and vice-versa. This can be visualized in Fig. 7. Taking into account the Fig. $7 \mathrm{~b}$ and signal D (Fig. 6a) as an example, the $\mathrm{B}$ input is the red $(\mathrm{R})$ channel and the $\sim \mathrm{B}$ input is the blue (B) one. The Invert input value 0 is the multiplexed signal under front illumination and the Invert value 1 is MUX signal under back illumination. The Invert selected as 0 results in the output of the sensor under front background, which is identical in a logic (on/off) point of view with the red signal in Fig. 6a and with the Green signal in Fig. 6b. The back line, which is the output when Invert is 1, is identical to the blue signal in Fig. $6 \mathrm{a}$ and the violet signal in Fig. 6b. This is the NOT function.

5.2 AND gate Several combinations are possible, the red-blue pair and the green-violet in Fig. 6 were chosen because the distance in wavelength within each pair is similar. We define these pairs as the input logical signals
D[R, B] (Fig. 6a) and P[G, V] (Fig. 6b). In Fig. 8 the multiplexed signal due to the combination of $\mathrm{P}$ and $\mathrm{D}$ digital signals is depicted. On the top the input signals guide the eyes into the ON/OFF states.

Under front and back irradiation, the $\mathrm{D}$ digital signal composed by the red and blue channels obeys to Eq. (3) where $\alpha_{\mathrm{R} ; \mathrm{B} \text {, Front }}^{\mathrm{V}}$ and $\alpha_{\mathrm{R} ; \mathrm{B} \text {, Back }}$ are respectively the optical gains under front and back violet background.

For the digital signal $\mathrm{P}$ with green and violet combination $\mathrm{P}[\mathrm{G}, \mathrm{V}]$ a similar equation results $\left[\mathrm{L}_{\mathrm{G}}(\mathrm{t}) \mathrm{S}_{\mathrm{V}}(\mathrm{t})\right]$.

$$
D=[R(t) 8(t)] \times\left[\begin{array}{ll}
\alpha_{\mathrm{R}, \text { Front }}^{\mathrm{V}} & \alpha_{\mathrm{R}, \text { Back }}^{\mathrm{V}} \\
\alpha_{\mathrm{B}, \text { Front }}^{\mathrm{V}} & \alpha_{\mathrm{B}, \text { Back }}^{\mathrm{V}}
\end{array}\right]=\left[L_{R}(t) S_{B}(t)\right]
$$

The $\mathrm{L}$ and $\mathrm{S}$ represent the signals in the long and shortwavelength range, respectively.

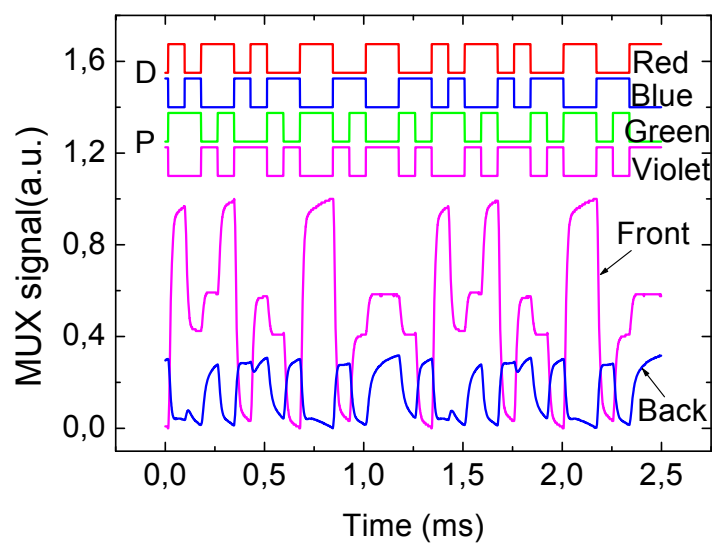

a)

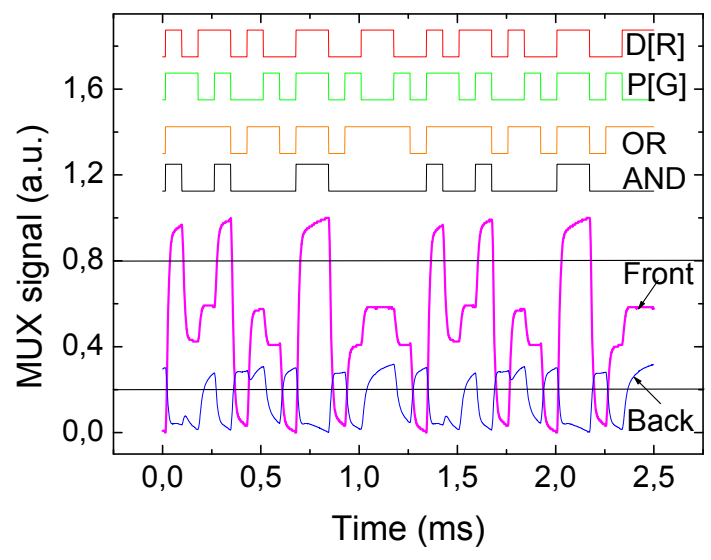

b)

Figure 8 Multiplexed signal due to the combination of P and D digital signals. On the top the signals used to drive the input channels are shown to guide the eyes.

The AND logical function (D AND P) is defined as having $T$ as output if and only if both inputs $\mathrm{D}, \mathrm{P}$ have value T. In Figure 8a under front irradiation it corresponds to both Red and Green channels on their ON state (the higher level under front illumination). So, it is possible to define a threshold line above which any result represents the D AND $\mathrm{P}$ output function. In our experimental conditions (Fig. 8b) this threshold could be around 0.8 cutting the 
multiplexed signal under front irradiation and yielding the and line which represents the AND output function to guide the eyes.

5.3 OR gate Another basic logic function is the OR. The OR logical function is defined (D OR P) with output T if either or both $\mathrm{D}, \mathrm{P}$ are $\mathrm{T}$ or equivalently the output is $\mathrm{F}$ if and only if both D, P are F. Taking into account the Fig. 6 and Fig. 8 it corresponds to the presence under front irradiation of the red, green or both channels in its ON state. This defines a threshold line above which any result is the D OR P function. In Fig $8 \mathrm{~b}$ this threshold could be around 0.2 cutting the front line and yielding the or line which represents the OR output function to guide the eyes.

5.4 XOR gate The OR function between two variables is true whenever either one or both are true. The logical function that only allows one of the variables to be true excludes the case of both being true. This is the exclusive or function also known as XOR. Figure $8 \mathrm{~b}$ shows the output function with the two threshold lines at 0.2 and 0.7 (a.u.). The OR function is identical to the signal being above the 0.2 line whilst the AND function is the signal being above the 0.7 line. To extract the XOR function the output signal must be between the two threshold lines yielding true as a result and false if the signal is outside those boundaries.

6 Conclusions A photonic pi'n/pin multilayered filter based on a-SiC:H technology is analyzed. Tailoring the wavelength was achieved by changing the violet background side. Results show that the structure becomes reconfigurable under front and back violet irradiation, acting either as data selector or data distributer. It performs WDM optoelectronic logic functions providing photonic functions such as signal amplification, filtering and switching.

A multiplexed signal, composed by four channels grouped into two inverted wavelength digital signals was analysed. The NOT function was shown and it imposed the base line of the digital signals. Other logical functions like the OR, AND, XOR were also identified using the same signals. More work has still to be done in order to execute optical logic and arithmetic operations entirely within the optical domain, based on $\mathrm{SiC}$ technology.

Acknowledgements This work was supported by FCT (CTS multi annual funding) through the PIDDAC Program funds and PTDC/EEA-ELC/111854/2009 and PTDC/EEAELC/120539/2010.

\section{References}

[1] M. Vieira, P. Louro, M. Fernandes, M. A. Vieira, A. Fantoni and J. Costa, Adv. Photodiodes, InTech, Chap. 19, pp. 403425 (2011).
[2] M. A. Vieira, M. Vieira, J. Costa, P. Louro, M. Fernandes, and A. Fantoni, Sens. Transduc. J. 9, 96-120 (2010) (Special Issue).

[3] M. G. Kuzyk, Polymer Fiber Optics, Materials Physics and Applications (Taylor and Francis Group, LLC, 2007).

[4] R. A. Street (ed.), Technology and Applications of Amorphous Silicon (Springer, New York, 2000), pp. 161-175.

[5] G. De Cesare, F. Irrera, F. Lemmi, F. Palma, and M. Tucci, a-Si:H/a-SiC:H heterostructure for bias-controlled photodetectors, MRS Proceedings, pp. 336, 885 doi:10.1557/PROC-336-885 (1994).

[6] W. Fuhs, Amorphous Hydrogenated Silicon, a-Si:H (Springer, New York, 2004), pp. 123-137.

[7] T. Neidlinger, R. Bruggemann, H. Brummak, and M. B. Schubert, J. Non-Cryst. Solids 227-230, 1335-1339 (1998).

[8] D. Caputo and G. Cesare, J. Non-Cryst. Solids 198-200, 1334-1336 (1996).

[9] D. Knipp, H. Stiebig, J. Fölsch, R. Carius, and H. Wagner, Mat. Res. Soc. Symp. Proc. 467, 931 (1997).

[10] H. Stiebig and M. Böhm, J. Non-Cryst. Solids. 164-166, 785 (1993).

[11] Z. Zhongwen, J. Multimedia Ubiq. Eng. 3(4), 17-24 (2008).

[12] R. Martins, G. Willeke, E. Fortunato, I. Ferreira, M. Vieira, M. Santos, A. Maçarico, and L. Guimarães. J. Non-Cryst. Solids 114, 486-488 (1989).

[13] P.P. Yupapin and P. Chunpang, Int. J. Light Electron. Opt. 120(18), 976-979 (2009).

[14] S. Ibrahim, L. W. Luo, S. S. Djordjevic, C. B. Poitras, I. Zhou, N. K. Fontaine, B. Guan, Z. Ding, K. Okamoto, M. Lipson, and S. J. B. Yoo, Optical Fiber Communications Conference, OSA/OFC/NFOEC, San Diego, 21 Mar. (2010), paper OWJ5.

[15] M. Bas, Fiber Optics Handbook, Fiber, Devices and Systems for Optical Communication (Mc Graw-Hill, Inc., 2002), Chap. 13.

[16] M.A. Vieira, P. Louro, M. Vieira, A. Fantoni, and A. Steiger-Garção. IEEE Sensor J. 12(6), 1755-1762 (2012).

[17] M. Vieira, P. Louro, M. A. Vieira, M. Fernandes, J. Costa, A. Fantoni, and M. Barata, Phys. Status Solidi C 7(3-4), 1184-187 (2010).

[18] S. Randel, A.M.J. Koonen, S.C.J. Lee, F. Breyer, M. Garcia Larrode, J. Yang, A. Ng'Oma, G.J Rijckenberg, and H.P.A. Boom. ECOC 07, Berlin, Germany, 2007 (Th 4.1.4) (pp. 14).

[19] T.A. Ibrahim et al., IEEE Photon. Technol. Lett. 15(10), 1422-1424 (2003).

[20] L. Zhang, J. Ding, Y. Tian, R. Ji, L. Yang, H. Chen, P. Zhou, Y. Lu, W. Zhu, and R. Min, Opt. Express 20(11),11605 (2012).

[21] M. Vieira, A. Fantoni, P. Louro, M. Fernandes, R. Schwarz, G. Lavareda, and C. N. Carvalho, Vacuum 82(12), 15121516 (2008).

[22] M. Vieira, A. Fantoni, P. Louro, M. Fernandes, R. Schwarz, G. Lavareda, and C. N. Carvalho, Vacuum 82(12), 15121516 (2008).

[23] M. A. Vieira, M. Vieira, V. Silva, P. Louro, and M. Barata MRS Proc. 1536, mrss13-1536-a19-06, doi:10.1557/opl.2013.703 (2013).

[24] V. Silva, M.A. Vieira, P. Louro, M. Vieira, and M. Barata, IFIP Adv. Inform. Commun. Technol. 394, 555-562 (2013). 